\title{
Glypican-3 promotes epithelial-mesenchymal transition of hepatocellular carcinoma cells through ERK signaling pathway
}

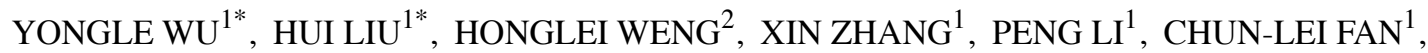 \\ BING LI ${ }^{1}$, PEI-LING DONG ${ }^{1}$, LEI LI ${ }^{1}$, STEVEN DOOLEY ${ }^{2}$ and HUI-GUO DING ${ }^{1}$ \\ ${ }^{1}$ Department of Gastroenterology and Hepatology, Beijing You'an Hospital affiliated with Capital Medical University, \\ Fengtai District, Beijing 100069, P.R. China; ${ }^{2}$ Molecular Hepatology, University of Heidelberg, \\ University Medical Center Mannheim, D-68167 Mannheim, Germany
}

Received October 20, 2014; Accepted December 10, 2014

DOI: 10.3892/ijo.2015.2827

\begin{abstract}
Glypican-3 (GPC3), a membrane-associated heparan sulfate proteoglycan, is frequently upregulated in hepatocellular carcinoma (HCC). However, how GPC3 contributes to the progress of HCC is largely unclear. The present study investigated the association between GPC 3 expression and HCC clinicopathological characteristics, and particularly focused on the role and underlying mechanisms of GPC3 in HCC epithelial-mesenchymal transition (EMT). Remarkably elevated expression of GPC 3 was demonstrated in HCC tumor tissues compared with paired non-tumor tissues in 45 patients with HCC by quantitative real-time PCR, immunohistochemistry, and western blotting, respectively. Furthermore, the tissue expression of GPC3 was increased during HCC progression from Barcelona Clinic Liver Cancer stage A or B to stage C. The enhanced levels of GPC3 in HCC tumor tissues were tightly correlated to the expression of the EMT-associated proteins and tumor vascular invasion. Patients with GPC3high expression in tumor tissues displayed significantly shorter survival time than those with GPC3-low expression $(\mathrm{P}=0.001)$. Consistent with the findings in patients, HepG2 cells, which expressed high levels of GPC3, showed stronger capacity of migration and significant EMT-like changes when compared to those HCC cells with low levels of GPC3, e.g., Hep3B
\end{abstract}

Correspondence to: Dr Hui-Guo Ding, Department of Gastroenterology and Hepatology, Beijing You'an Hospital affiliated with Capital Medical University, Fengtai District, Beijing 100069, P.R. China

E-mail: dinghuiguo@medmail.com.cn

*Contributed equally

Abbreviations: GPC3, glypican-3; HCC, hepatocellular carcinoma; EMT, epithelial-mesenchymal transition; ERK, extracellular signalregulated kinase; GPI, glycosyl-phosphatidylinositol; AFP, $\alpha$-fetoprotein; BCLC, Barcelona Clinic Liver Cancer; MAPK, mitogenactivated protein kinas; Shh, Sonic hedgehog

Key words: glypican-3, hepatocellular carcinoma, metastasis, invasion, epithelial-mesenchymal transition, extracellular signal-regulated kinase and Huh7 in scratch, Transwell assays and western blotting. Furthermore, administration with exogenous GPC3 in HCC cells activated extracellular signal-regulated kinase (ERK) and significantly enhanced cell migration and invasion. The behavior was significantly inhibited by the ERK inhibitor PD98059. Together, our studies show that GPC3 contributes to HCC progression and metastasis through impacting EMT of cancer cells, and the effects of GPC3 are associated with ERK activation.

\section{Introduction}

Hepatocellular carcinoma (HCC) is one of the most common cancers, and the third leading cause of cancer-related mortality worldwide (1). The main risk factors of HCC in humans include infection with hepatitis $\mathrm{B}$ and/or hepatitis $\mathrm{C}$ virus, and alcoholic liver disease (2). The outcome of HCC treatment is dependent on the tumor stage at the time of diagnosis. A good curability can be achieved if HCC were detected in an early stage. Unfortunately, most HCC patients with symptoms have progress to advanced stage when they are diagnosed. The poor prognostic outcomes are mainly due to cancer metastasis and tumor recurrence after surgery. Various factors are involved in the regulation of cancer metastasis, including the epithelial-mesenchymal transition (EMT) (3). Nevertheless, the molecular mechanisms underlying EMT in HCC tumor metastasis are poorly characterized.

Glypican-3 (GPC3) is one of the cell surface heparan sulfate proteoglycans that bind to the cell membrane via a glycosyl-phosphatidylinositol (GPI) anchor. GPC3 is released from cell membranes by the extracellular lipase Notum at the GPI anchor (4). Functionally, GPC3 regulates signaling pathways associated with the Wnt, Sonic hedgehog (Shh), fibroblast growth factor, and bone morphogenetic protein (5-7). Previous studies indicated that GPC3 plays an important role in regulating cancer progression in a stage- and tissue-specific manner; e.g. GPC3 acted as a tumor suppressor in breast cancer $(7,8)$. Interestingly, overexpression of GPC3 presented in lung squamous cell carcinoma, but not in lung adenocarcinoma (9).

GPC3 is involved in the migration, proliferation, and modulation of cell survival in different tissues (8). It has been shown 
that GPC3 is highly expressed in patients with $\mathrm{HCC}$, either in serum or liver tissues, but is absent in benign hepatic lesions, hepatic cirrhosis and hepatitis (10-12). Furthermore, GPC3 has been reported to be used to discriminate $\alpha$-fetoprotein (AFP)negative HCC from cirrhotic nodules (13). Thus, GPC3 might be a more reliable tumor marker that could allow for an early diagnosis of HCC $(14,15)$. In addition, several studies have shown that GPC3 promoted growth of $\mathrm{HCC}$ in vivo and in vitro $(6,16,17)$. However, the clinical function of GPC3 in HCC progression remains obscure. To date, few studies elucidate the potential functions of GPC3 in HCC EMT and metastasis.

The present study examined the effects of GPC3 in HCC progression and metastasis. We focused on the role of GPC3 in HCC EMT and underlying molecular mechanisms.

\section{Materials and methods}

Patients. We obtained paired liver tissues (tumor vs. non-tumor) from 45 patients with HCC who received hepatic resection between July 2008 and January 2009 at Beijing You'an Hospital. One part of resected tissues was fixed in formalin and embedded in paraffin for histological examination. The remained liver tissues were snap-frozen and stored at $-80^{\circ} \mathrm{C}$ for real-time PCR and western blot analyses. Clinical characteristics of these patients are shown in Table I. HCC was confirmed by histological examinations according to the AASLD guidelines proposed in 2005 (18). This study was approved by Research Ethics Committee, You'an Hospital affiliated with Capital Medical University. Written informed consent was obtained from each patient.

Immunohistochemistry analyses for GPC3. Four- $\mu \mathrm{m}$ paraffinembedded sections were deparaffinized and rehydrated. Antigen was retrieved using heat-induced epitopes in $10 \mathrm{mM}$ citrate buffer, $\mathrm{pH} 6.0$ and endogenous peroxidases were blocked for 5 min using $0.3 \% \mathrm{H}_{2} \mathrm{O}_{2}$. Then, the sections were incubated with mouse monoclonal anti-GPC3 antibody (diluted 1:100, Santa Cruz Biotechnology, Santa Cruz, CA, USA) overnight in a cool room. Next day, the slides were applied for horseradish peroxidase-labeled secondary antibody (Zhongshan Golden Bridge, Beijing, China) after three PBS washes. To evaluate GPC3 immunostaining, the percentage of positive cells and intensity of staining were analyzed. The intensity of immunostaining was semi-quantitatively estimated according to the following scores: 0 , no positive staining; 1 , positive staining was weak yellow; 2, positive staining was yellow; and 3, strong positive staining was brown. The mean percentage of positive tumor cells was determined after counting positive GPC3 cells in five fields under x400 magnification. The scores were defined as follows: $0, \leq 10 \% ; 1,10 \%-25 \% ; 2,25 \%-50 \%$; $3,50-75 \%$; and $4,>75 \%$. The final GPC3 immune score was calculated as staining intensity $\mathrm{x}$ mean percentage of positive tumor cells. We defined the scores $\leq 4$ as low expression of GCP3 and 5-12 as high expression of GPC3.

RNA extraction and quantitative real-time PCR. Total RNA was purified from frozen HCC tissues using an RNeasy Mini kit (Qiagen, Hilden, Germany). Total RNA (1 $\mu \mathrm{g})$ was reverse transcribed using a Reverse-Transcription system (Promega, Madison, WI, USA). Transcript levels of GPC3 cDNA were
Table I. Clinical characteristics of the study population $(n=45)$.

$\begin{array}{lc}\text { Age (mean/range) (years) } & 48.9(22-67) \\ \text { Gender (male/female) } & 38 / 7 \\ \text { Primary liver disease } & \\ \text { (HBV/HCV/alcohol/others) } & 39 / 3 / 4 / 2 \\ \text { Patients with cirrhosis } & 45 \\ \text { Child-Pugh class }(\mathrm{A} / \mathrm{B} / \mathrm{C}) & 27 / 18 / 0 \\ \text { Tumor diameter }(<3 \mathrm{~cm} / \geq 3 \mathrm{~cm}) & 14 / 31 \\ \text { Tumor number }(\leq 3 />3) & 40 / 5 \\ \text { Portal vein thrombosis } & 14 \\ \text { Vascular invasion (present/absent) } & 14 / 31 \\ \text { Serum AFP ( } \leq 20 \mu \mathrm{g} / \mathrm{l} />20 \mu \mathrm{g} / \mathrm{l}) & 19 / 16 \\ \text { Histological differentiation } & \\ \text { (good/moderate and poor) } & 4 / 18 / 23 \\ \text { BCLC stage (A/B/C/D) } & 13 / 14 / 18 / 0\end{array}$

$\mathrm{HBV}$, hepatitis B virus; $\mathrm{HCV}$, hepatitis $\mathrm{C}$ virus; $\mathrm{AFP}, \alpha$-fetoprotein; BCLC, the Barcelona Clinic Liver Cancer.

quantified using real-time PCR (Rotor-Gene Q, Qiagen, Germany). The primers used to amplify GPC3 were: forward, 5'-TTCTCAACAACGCCAATA-3', and reverse, 5'-GATGTA GCCAGGCAAAGC-3'. Amplicon expression in each sample was normalized to $\beta$-actin. The primers used to amplify $\beta$-actin were: forward, 5'-ACCCACACTGTGCCCATCTA-3', and reverse, 5'-GCCACAGGATTCCATACCCA-3'. After normalization, expression of GPC3 was quantified using a $2^{-\Delta \Delta C t}$ calculation. PCR was performed in duplicate and three independent experiments were performed.

Cell culture. The human HCC cell lines HepG2, Hep3B and Huh7 were obtained from the Cell Bank of the Type Culture Collection of the Chinese Academy of Sciences (Shanghai, China). HepG2, Hep3B and Huh7 were cultured in Dulbecco's modified Eagle's medium (DMEM; Gibco Life Technology, Carlsbad, CA, USA) supplemented with $10 \%$ fetal bovine serum (FBS; Hyclone, Rockford, IL, USA), $100 \mu \mathrm{g} / \mathrm{ml}$ streptomycin, and $100 \mathrm{U} / \mathrm{ml}$ penicillin (Beijing Solarbio Science \& Technology Co., Ltd., Beijing, China) in a humidified atmosphere of $5 \% \mathrm{CO}_{2}$ at $37^{\circ} \mathrm{C}$. For experiments, cells at $70 \%$ confluence were serum-starved $24 \mathrm{~h}$ before treatment. To block the ERK pathway, cells were preincubated with PD98059 (Sigma-Aldrich, St. Louis, MO, USA) for $3 \mathrm{~h}$ before GPC3 (Sino Biological Inc., Beijing, China) treatment.

Western blotting. Western blotting was performed as described previously (19). Briefly, total cellular proteins were extracted using cell lysis buffer $(20 \mathrm{mM}$ Tris/ $\mathrm{HCl}, 150 \mathrm{mM} \mathrm{NaCl}$, $1 \%$ Nonidet P-40, 0.1\% SDS and protease inhibitor cocktail, $\mathrm{pH}$ 7.5). The protein concentrations of the lysates were determined according to the bicinchoninic acid (BCA) method using a protein assay kit (Pierce Biotechnology, Rockford, IL, USA). Cell or liver tissue lysates containing $100 \mu \mathrm{g}$ protein were generated and total proteins were separated by standard 
SDS-PAGE, followed by transfer to polyvinylidene difluoride membranes (Millipore, Billerica, MA, USA). Membranes were incubated with primary antibodies against GPC3 (diluted 1:500, Santa Cruz Biotechnology), MMP9 (diluted 1:1,000, Cell Signaling Technology, CA, USA), E-cadherin (diluted 1:1000, Santa Cruz Biotechnology), $\alpha$-SMA (diluted 1:1,000, Cell Signaling Technology), $\beta$-catenin (diluted 1:1,000, Cell Signaling Technology), Snail (diluted 1:1,000, Cell Signaling Technology), p-ERK (diluted 1:1,000, Cell Signaling Technology), ERK (diluted 1:1,000, Cell Signaling Technology), or Sonic hedgehog (Shh; diluted 1:500, Santa Cruz Biotechnology). Next, membranes were incubated with an appropriate horseradish peroxidase-conjugated secondary antibody. Reactive bands were detected using enhanced chemiluminescence reagents (Applygen Technologies Inc., Beijing, China). To ensure equal loading of samples in each lane, membranes were stripped and reprobed with an anti-glyceraldehyde 3-phosphate dehydrogenase antibody (GAPDH; diluted 1:10,000; Kang Chen, Shanghai, China). The relative densities of the protein bands were quantitatively determined using Image J software (National Institutes of Health, Bethesda, MD, USA).

Scratch assay. The migration of cells was examined with scratch assays. When cells grew to $90 \%$ confluency, a scratch wound in the monolayer was made using a pipette tip. After washing away all detached cells with PBS, the remaining cells were treated with $0.1 \mu \mathrm{g} / \mathrm{ml}$ GPC 3 . The wound distances were measured by microscope at 0,24 , and $48 \mathrm{~h}$ after treatment. Each test was performed in triplicate.

Migration and invasion assays. Serum-starved cells were resuspended in DMEM free of FBS and seeded in a 24-well plate at a concentration of $10^{5}$ cells/well on inserts with $8-\mu \mathrm{m}$ filter pores, which were either uncoated (migration assay; BD Falcon $^{\text {TM }}$ Cell Culture Insert, San Jose, CA, USA) or Matrigelcoated (invasion assay; BD Biocoat ${ }^{\mathrm{TM}}$ Matrigel $^{\mathrm{TM}}$ Invasion Chamber). As a chemoattractant, $1 \%$ FBS was added to the lower chamber while FBS-free DMEM with 0, 0.05, 0.1 or $0.2 \mu \mathrm{g} / \mathrm{ml}$ GPC3 or $0,25,50$ or $100 \mathrm{nM}$ PD98059 was put into the upper chamber, respectively. After $24 \mathrm{~h}$, cells were fixed and stained with methanol and hematoxylin. Migrated or invaded cells were counted at ten random fields per triplicate filter under an inverted microscope whereas non-migrating and non-invading cells were removed from the upper surface by wiping with a cotton swab. Each test was performed in triplicate.

Statistical analysis. Clinicopathological parameters in patients with GPC3 high/low were compared using Pearson's $\chi^{2}$ test or Fisher's exact test. Continuous variables were analyzed with one-way analysis of variance. The results of multiple observations are presented as the means \pm SDs of at least three independent experiments. The Kaplan-Meier method was used to determine survival probability and differences between groups were assessed using the log-rank test. Overall survival (OS) was defined as the interval between surgery and death or the last follow-up time-point. Statistical analyses were conducted using SPSS version 17.0 software (SPSS Inc., Chicago, IL, USA).
Table II. The correlation between GPC3 expression and clinicopathologic variables of patients with HCC. ${ }^{\text {a }}$

\begin{tabular}{|c|c|c|c|}
\hline Feature & $\begin{array}{c}\text { Low } \\
\text { expression } \\
\text { of GPC3 } \\
(n=23)\end{array}$ & $\begin{array}{c}\text { High } \\
\text { expression } \\
\text { of GPC3 } \\
(n=22)\end{array}$ & P-value \\
\hline \multicolumn{4}{|l|}{ Gender } \\
\hline Male & 19 & 19 & \multirow[t]{2}{*}{1.000} \\
\hline Female & 4 & 3 & \\
\hline \multicolumn{4}{|l|}{ Age (years) } \\
\hline$\leq 50$ & 10 & 13 & \multirow[t]{2}{*}{0.295} \\
\hline$>50$ & 13 & 9 & \\
\hline \multicolumn{4}{|l|}{ HBsAg } \\
\hline Positive & 21 & 18 & \multirow[t]{2}{*}{0.619} \\
\hline Negative & 2 & 4 & \\
\hline \multicolumn{4}{|c|}{ Child-Pugh class } \\
\hline A & 15 & 12 & \multirow[t]{2}{*}{0.465} \\
\hline $\mathrm{B}$ & 8 & 10 & \\
\hline \multicolumn{4}{|c|}{ Tumor size $(\mathrm{cm})$} \\
\hline$\leq 3$ & 7 & 7 & \multirow[t]{2}{*}{0.920} \\
\hline$>3$ & 16 & 15 & \\
\hline \multicolumn{4}{|c|}{ Tumor number } \\
\hline Single & 14 & 17 & \multirow[t]{2}{*}{0.235} \\
\hline Multiple & 9 & 5 & \\
\hline \multicolumn{4}{|c|}{ Vascular invasion } \\
\hline No & 22 & 9 & \multirow[t]{2}{*}{0.001} \\
\hline Yes & 1 & 13 & \\
\hline \multicolumn{4}{|c|}{ Serum AFP $(\mu \mathrm{g} / \mathrm{l})$} \\
\hline$\leq 20$ & 10 & 9 & \multirow[t]{2}{*}{0.862} \\
\hline$>20$ & 13 & 13 & \\
\hline \multicolumn{4}{|c|}{ BCLC HCC stage } \\
\hline $\mathrm{A}$ & 9 & 4 & $0.002^{\mathrm{b}}$ \\
\hline $\mathrm{B}$ & 11 & 3 & \multirow[t]{2}{*}{$0.001^{\mathrm{b}}$} \\
\hline $\mathrm{C}$ & 3 & 15 & \\
\hline
\end{tabular}

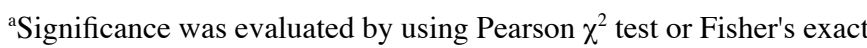
test. ${ }^{b}$ Compared with BCLC stage C. AFP, $\alpha$-fetoprotein; HBsAg, hepatitis B surface antigen; BCLC, Barcelona Clinic Liver Cancer; $\mathrm{HCC}$, hepatocellular carcinoma.

\section{Results}

High expression of GPC3 in cancer cells is associated with HCC progression. To examine whether GPC3 involved in HCC progression, GPC 3 mRNA and protein levels were assessed in liver tissues from patients with different HCC stages. The Barcelona Clinic Liver Cancer (BCLC) system was applied to classify the disease stage (20). Among the 45 pathologically diagnosed HCC patients, 13 had BCLC-stage A, 14 BCLCstage $\mathrm{B}$, and 18 BCLC-stage $\mathrm{C}$. The expression of GPC3 was examined in all HCC tissues using an anti-GPC3 antibody. Immunostaining of GPC3 was detected in the majority of hepatocyte membranes and cytoplasms of most HCC cancer cells (Fig. 1A). Notably, both mRNA and protein expression of 



Figure 1. GPC3 expression correlates with HCC progression. (A) Relative expression of GPC 3 in 45 HCC patients determined by immunohistochemical staining was significantly elevated in HCC patients with BCLC-stage C compared to those with BCLC-stage A or B (original magnification, x100). (B) Representative GPC3 expression in $45 \mathrm{HCC}$ cancerous and paracancerous tissues were determined by western blotting. (C) We measured GPC3 mRNA expression levels normalized to $\beta$-actin levels in all cancerous and paracancerous tissues by quantitative real-time PCR. (D) Correlation of GPC3 expression with the overall survival rates of patients. BCLC, Barcelona Clinic Liver Cancer; C, cancerous liver tissues; P, paracancerous liver tissues. ${ }^{*} \mathrm{P}<0.01$.

GPC3 was remarkably elevated in HCC patients with BCLCstage C compared to those with BCLC-stage A or B (Fig. 1B and $\mathrm{C})$. Real-time PCR and western blot analyses showed that $G P C 3$ was upregulated on average $\leq 2.4$-fold in patients with BCLC-stage C compared to those with BCLC-stage A or B $(\mathrm{P}=0.003$, Fig. $1 \mathrm{~B}$ and $\mathrm{C})$. There were no significant differ- ence of GPC3 expression between patients with BCLC-stages $\mathrm{A}$ and $\mathrm{B}(\mathrm{P}>0.05$; Fig. $1 \mathrm{~B}$ and $\mathrm{C})$, or the paracancerous tissues of different stages $(\mathrm{P}>0.05$; Fig. 1B and $\mathrm{C})$.

As mentioned in Materials and methods, we divided 45 patients into $\mathrm{GPC}^{\text {high }}$ and $\mathrm{GPC} 3^{\text {low }}$ groups based on GPC 3 immune positivity. Table II shows that 22 patients 

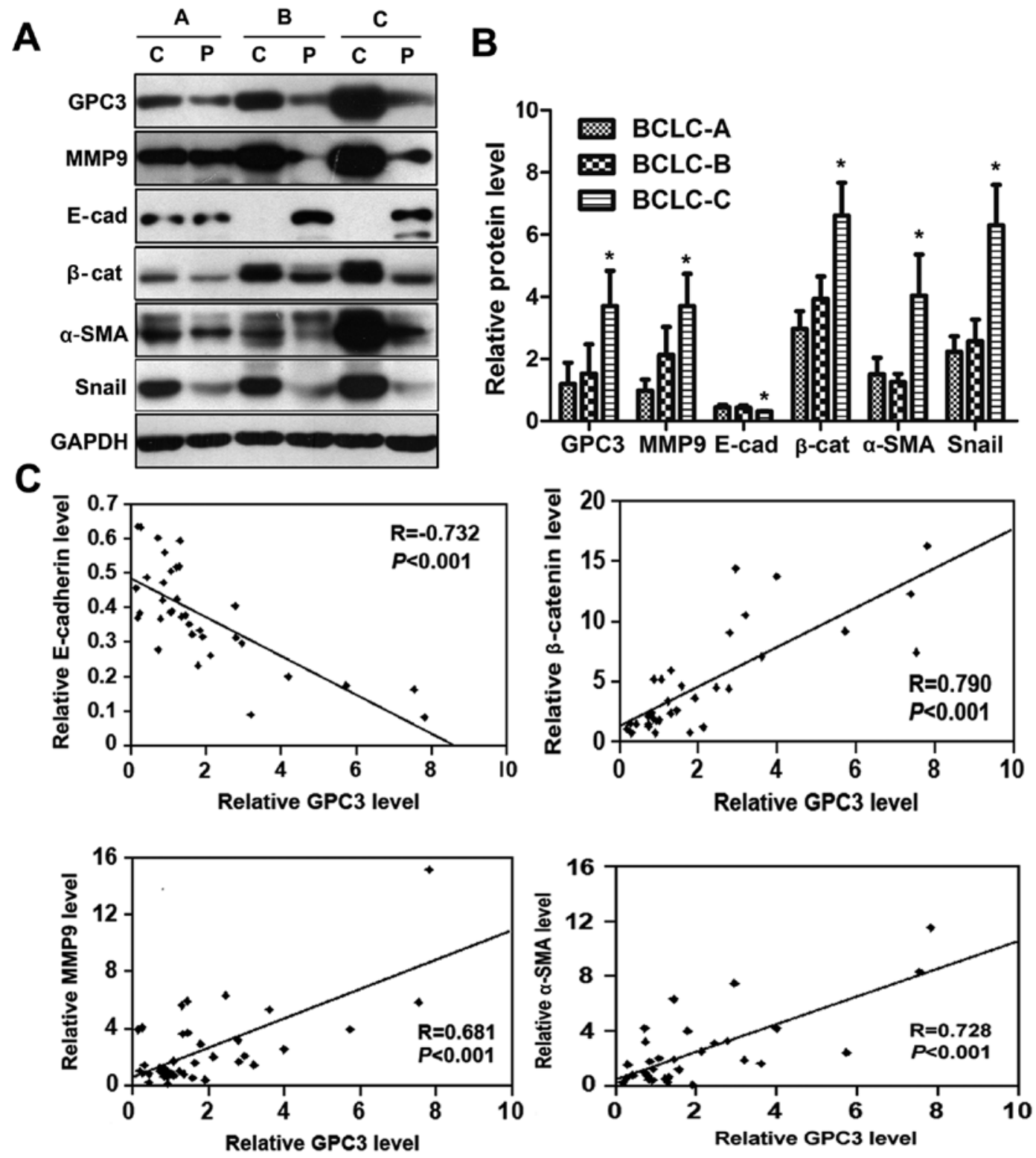

Figure 2. Correlation of GPC3 expression with invasion-associated proteins. (A and B) Representative MMP9, E-cadherin, $\beta$-catenin, $\alpha$-SMA and Snail expression in tissues with different BCLC stages. Data are shown as means \pm SDs of four independent experiments (B). ${ }^{*} \mathrm{P}<0.05$. (C) The correlation of MMP9, E-cadherin, $\beta$-catenin, and $\alpha$-SMA with GPC3 expression in HCC biopsies.

(48.9\%) belonged to GPC3 ${ }^{\text {high }}$ group and $23(51.1 \%)$ GPC3 ${ }^{\text {low }}$ group, respectively. Correlations between GPC3 expression and clinicopathological features were further analyzed. Consistent with the findings based on real-time PCR and western blotting, there was a significant difference in GPC3 expression levels between BCLC-stages $\mathrm{C}$ and $\mathrm{A}(\mathrm{P}=0.002)$ or $\mathrm{B}(\mathrm{P}=0.001)$, while there was no significant difference between BCLC-stages A and B. In addition, high levels of GPC3 expression were strongly correlated with the rate of vascular invasion $(\mathrm{P}<0.001)$. No other clinical characteristics, including age, gender, hepatitis B virus infection, serum AFP level, tumor size, tumor number, and Child-Pugh class, were related to the expression of GPC 3 . We then investigated whether the GPC3 expression is associated with the prognosis of HCC patients. The 5-year overall survival rate of HCC patients with low GPC3 expression levels reached $80.8 \%$ whereas only $31.8 \%$ of the HCC patients with high GPC3 expression were still surviving at the end of the follow-up ( $\mathrm{P}=0.042$, Mantel-Cox test; Fig. 1D).

HCC patients with high GPC3 expression demonstrated the EMT phenotype. HCC progression and metastasis were highly associated with phenotypic alterations of cancer cells, particularly EMT $(21,22)$. In this study, we measured the expression of the epithelial cell markers, e.g., E-cadherin and $\beta$-catenin, and the mesenchymal markers such as MMP9 and $\alpha$-SMA, as well as Snail, a transcription factors associated with EMT. Western blot analyses showed that the expression of Snail, MMP9, $\beta$-catenin and $\alpha$-SMA in cancerous tissues with BCLC-stage $\mathrm{C}$ were significantly increased compared to those with BCLC-stages A or B ( $\mathrm{P}<0.05$, Fig. 2A). By contrast, expression of E-cadherin in cancerous tissues with BCLCstage $\mathrm{C}$ was lower than in those with BCLC-stages $\mathrm{A}$ or $\mathrm{B}$ $(\mathrm{P}<0.05$; Fig. 2A). There was a positive correlation between the 
A
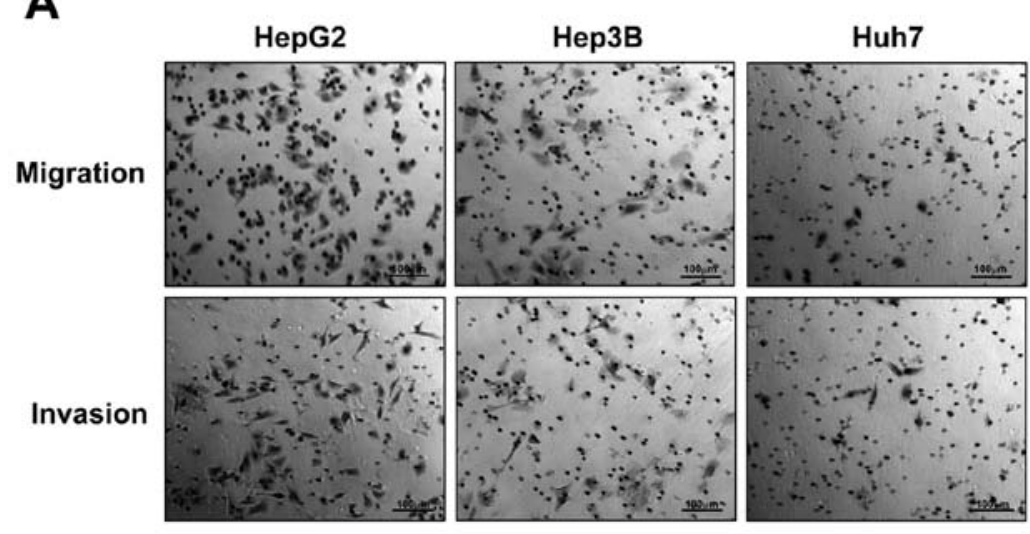

B

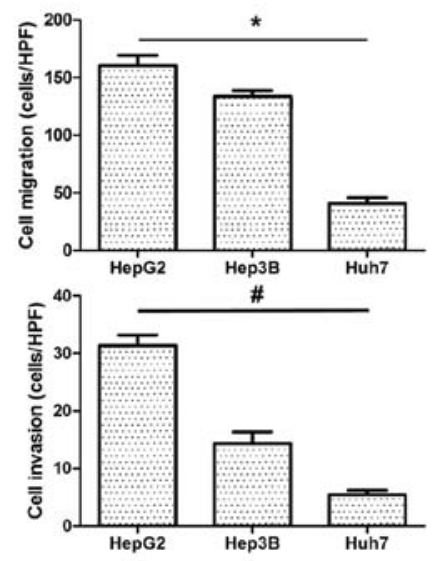

C
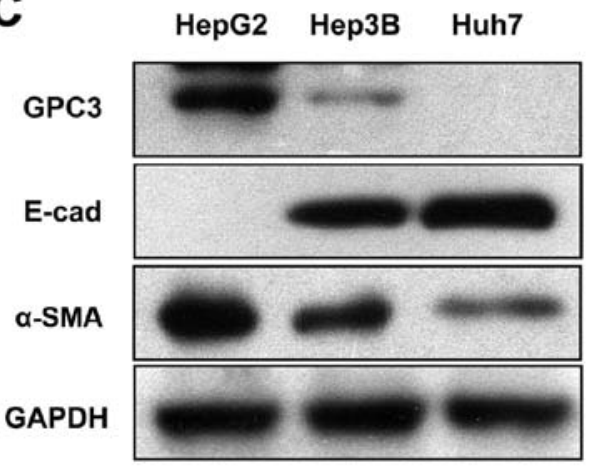

D

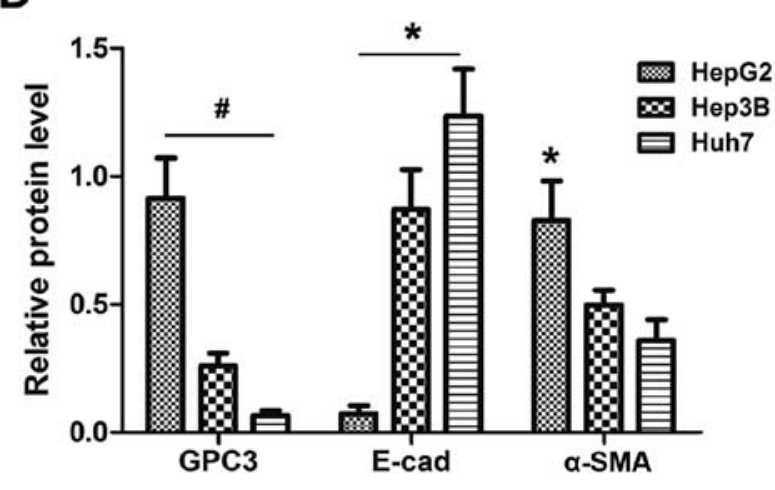

Figure 3. Expression of GPC3 was associated with the migration and invasion capacities of HCC cells. (A) Transwell assays to measure the migration (upper) and invasion (lower) of HepG2, Hep3B, and Huh7 cell lines. (B) Bar charts of migrated (upper) and invaded (lower) cells for each HCC cell line. Data are shown as means \pm SDs, ${ }^{*} \mathrm{P}<0.05$ and ${ }^{*} \mathrm{P}<0.01$. $\mathrm{n}=4$. (C) The expression of GPC3 and EMT markers in each HCC cell line. (D) Densitometric analysis of protein levels are shown in the diagram. Data are shown as means $\pm \mathrm{SDs},{ }^{*} \mathrm{P}<0.05$ and ${ }^{\#} \mathrm{P}<0.01$. $\mathrm{n}=3$.

expression levels of GPC3 and MMP9, $\beta$-catenin, and $\alpha$-SMA, but a negative correlation between the expression of GPC3 and E-cadherin (Fig. 2B). These results strongly suggest that GPC3 might contribute to EMT of HCC.

GPC3 participates in EMT in vitro. To further clarify the potential association between GPC3 expression and HCC metastasis, we investigated the expression of GPC3 and the metastatic capacity in HCC cell lines e.g., HepG2, Hep3B and Huh7. Migration and invasion assays indicated that HepG2 cells had the highest and Huh7 cells had the lowest metastatic capacity among three HCC cell lines ( $\mathrm{P}<0.05$; Fig. 3A). Western blotting showed that HepG2 cells expressed the highest levels of GPC3, whereas Huh7 cells expressed the lowest (Fig. 3C). Alternatively, HepG2 cells expressed higher levels of $\alpha$-SMA and lower levels of E-cadherin compared to Hep3B and Huh7 cells (Fig. 3C and D).

In addition to the cell surface expression, GPC3 can also be detected in the extracellular environment after being released from the cell membrane by Notum, a lipase that cleaves GPI anchors (4). Next, the impact of soluble exogenous GPC3 on HCC cellular motility was studied. Scratch-assays displayed an obviously greater migration capacity of both HepG2 and Hep3B cells when they were treated by exogenous GPC3 compared to untreated cells (Fig. 4A). Additionally, in Boyden chamber and Matrigel invasion assays, exogenous GPC3 induced dynamic migration and invasion of both HepG2 and Hep3B cells in a dose-dependent manner (Fig. 4B and C). Furthermore, GPC3 treatment significantly reduced expression of the epithelial cell markers such as E-cadherin and increased the mesenchymal markers MMP9 and $\alpha$-SMA in a dose-dependent manner in both HepG2 and Hep3B cells.

GPC3 induces EMT via increasing ERK activation. Given the critical roles of the mitogen-activated protein kinase (MAPK) (23) and Sonic hedgehog (Shh) signaling pathways in EMT (24), we investigated the impact of GPC3 in the two pathways. We found that ERK activity was strongly elevated in HCC patients with BCLC-stage $\mathrm{C}(\mathrm{P}<0.05$; Fig. 5A). A positive correlation between GPC3 expression and phospho-ERK was observed in HCC tissues by regression analysis, with a coefficient $(\mathrm{R})$ of $0.803(\mathrm{P}<0.001$; Fig. 5B). In vitro, the exposure of cells to soluble GPC3 promoted the phosphorylation of ERK in a dose-dependent manner in both HepG2 and Hep3B cells (Fig. 5C). To further confirm whether GPC3-induced cell migration and invasion is via the ERK pathway, we applied PD98059, an inhibitor of MEK (a kinase that activates ERK), to HepG2 cells prior to GPC3 treatment. We observed that PD98059 could decrease HepG2 cell migration and invasion. Furthermore, this inhibitor significantly attenuated the GPC3-induced migration of HepG2 cells in a dose-dependent

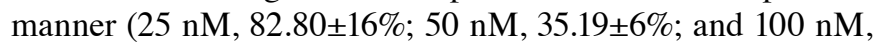


A

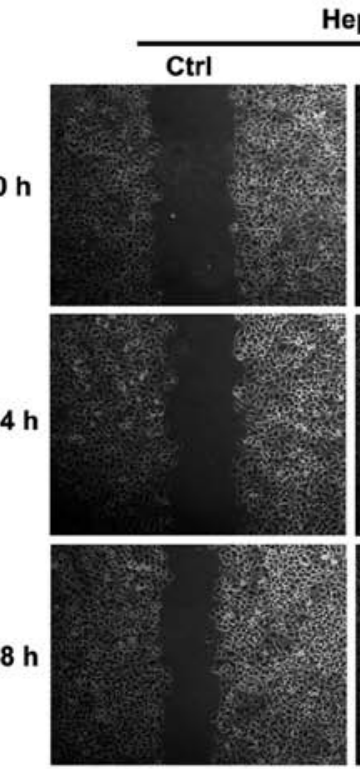

HepG2

B

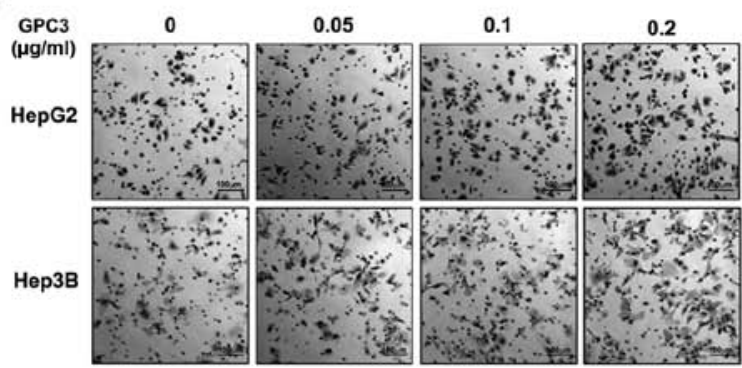

GPC3
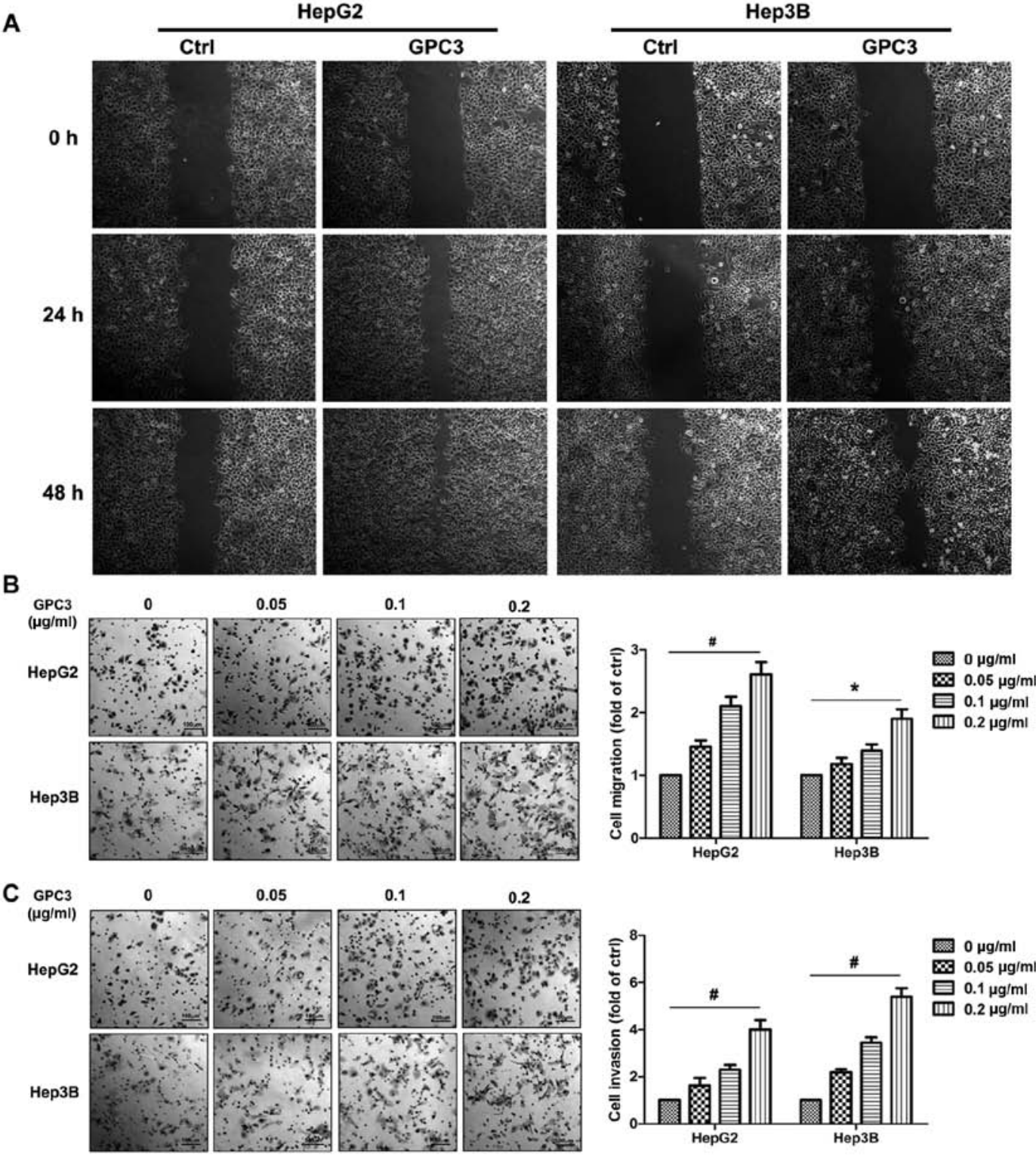

D
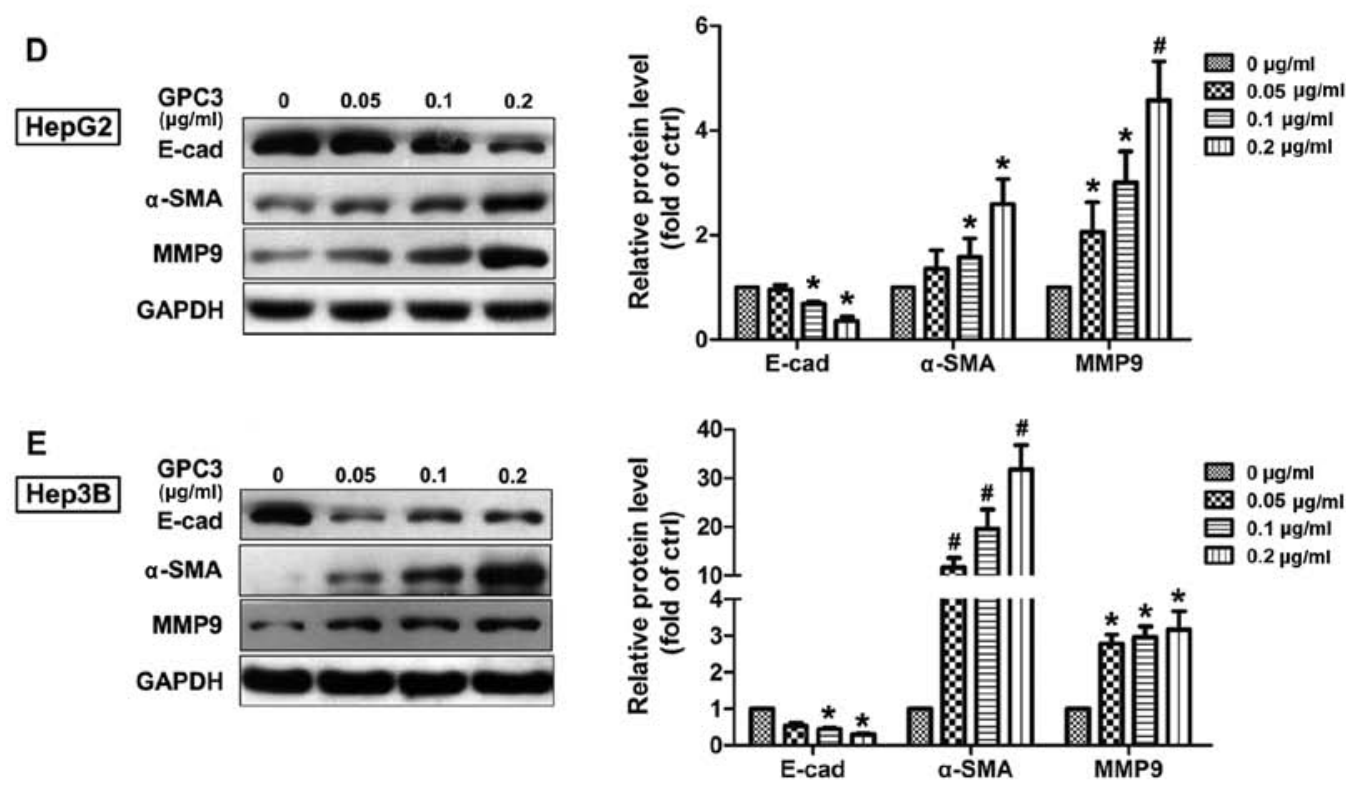

Figure 4. Exogenous GPC3 enhanced the migration and invasion of HCC cells. (A) The migration of HCC cells treated with exogenous GPC 3 were detected by scratch assays (magnification, x200). (B and C) Exogenous GPC3 increased the migration (B) and invasion (C) capacity of HCC cells in Transwell assays. (D and E) The effect of exogenous GPC3 on EMT markers in HepG2 (D) and Hep3B (E) cells. Protein expression levels were normalized to GAPDH and are expressed as the fold of control. Untreated cells served as controls in all panels. Data are shown as means \pm SDs ( $\mathrm{P}<0.05$ and $\left.{ }^{\#} \mathrm{P}<0.01\right)$. $\mathrm{n}=3$ (B and $\mathrm{C}$ ); $\mathrm{n}=4$ (D and E). 
A

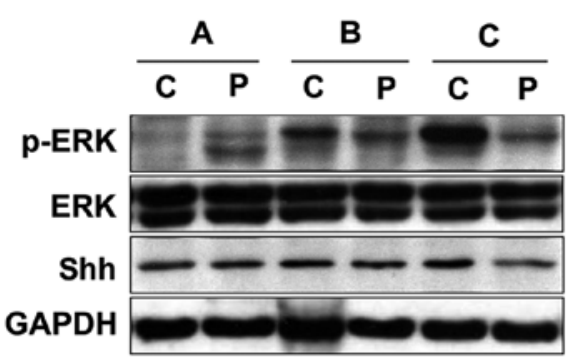

B

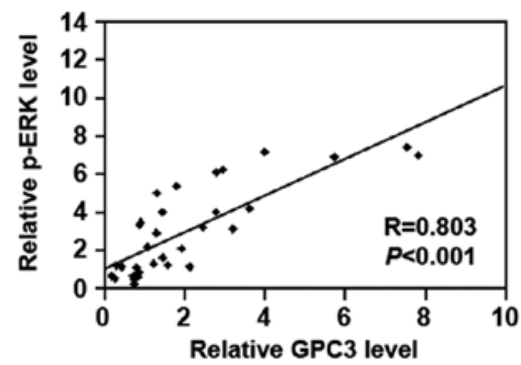

C
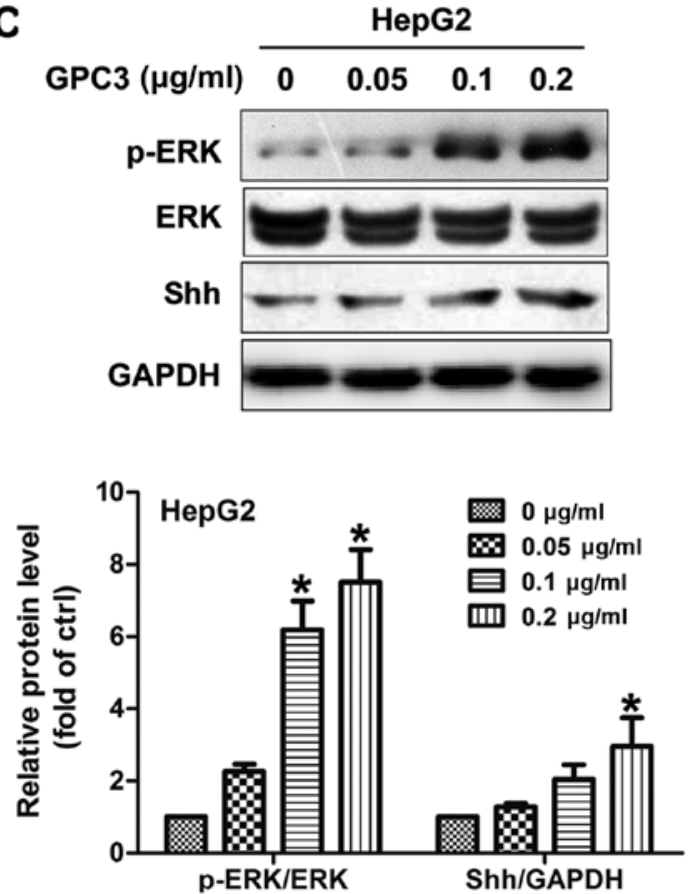
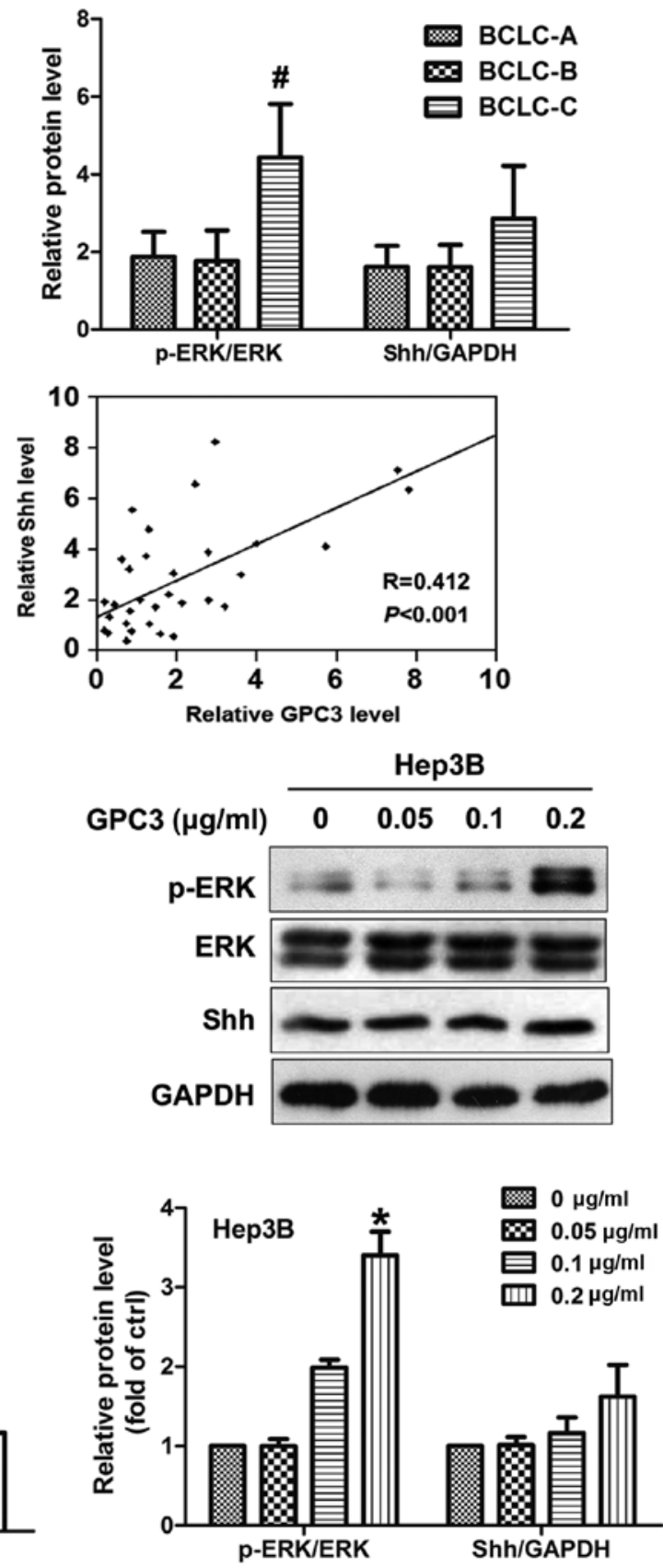

Figure 5. ERK activation may be regulated by GPC3. (A) ERK activity and Shh expression were correlated with HCC progression. (B) Correlation of GPC3 expression with ERK activation and Shh expression in HCC tissues, respectively. (C) The effects of soluble GPC3 treatment on ERK activity and Shh expression in HCC cells. Untreated cells were used as controls. Cells treated only with GPC3 served as controls. Data are shown as means \pm SDs based on three independent experiments; ${ }^{*} \mathrm{P}<0.05$ and ${ }^{\#} \mathrm{P}<0.01$ vs. control cells.

$24.32 \pm 6 \%$ ). In an invasion assay, PD98059 reduced the GPC3induced invasion capacity of HepG2 cells in a dose-dependent manner $(25 \mathrm{nM}, 73.00 \pm 6.74 \%$; $50 \mathrm{nM}, 41.70 \pm 3.07 \%$; and $100 \mathrm{nM}, 35.54 \pm 2.13 \%$; Fig. 6A). Notably, PD98059 significantly attenuated GPC3-induced EMT. As shown in Fig. 6B, E-cadherin was increased whereas MMP-9, $\alpha$-SMA and Snail were decreased under PD98059 treatment. Besides the ERK pathway, we also investigated whether the Shh pathway was involved in GPC3-induced EMT. Unexpectedly, no significance was obtained on Shh expression among different stages ( $\mathrm{P}>0.05$; Fig. 5A). The correlation of Shh expression with GPC3 was weaker compared to ERK (Fig. 5B). In vitro, GPC3 treatment could not markedly increase the Shh expression (Fig. 5C).

\section{Discussion}

The expression levels of GPC3 mRNA and protein were markedly increased in primary tissues and serum from HCC patients compared to those from healthy people or patients with benign liver lesions $(10,12,13)$. However, the clinical role of GPC3 in HCC progression and the underlying molecular mechanisms have remained unclear. Here, we showed that GPC 3 expression was correlated with the clinical features of $\mathrm{HCC}$, including 
A
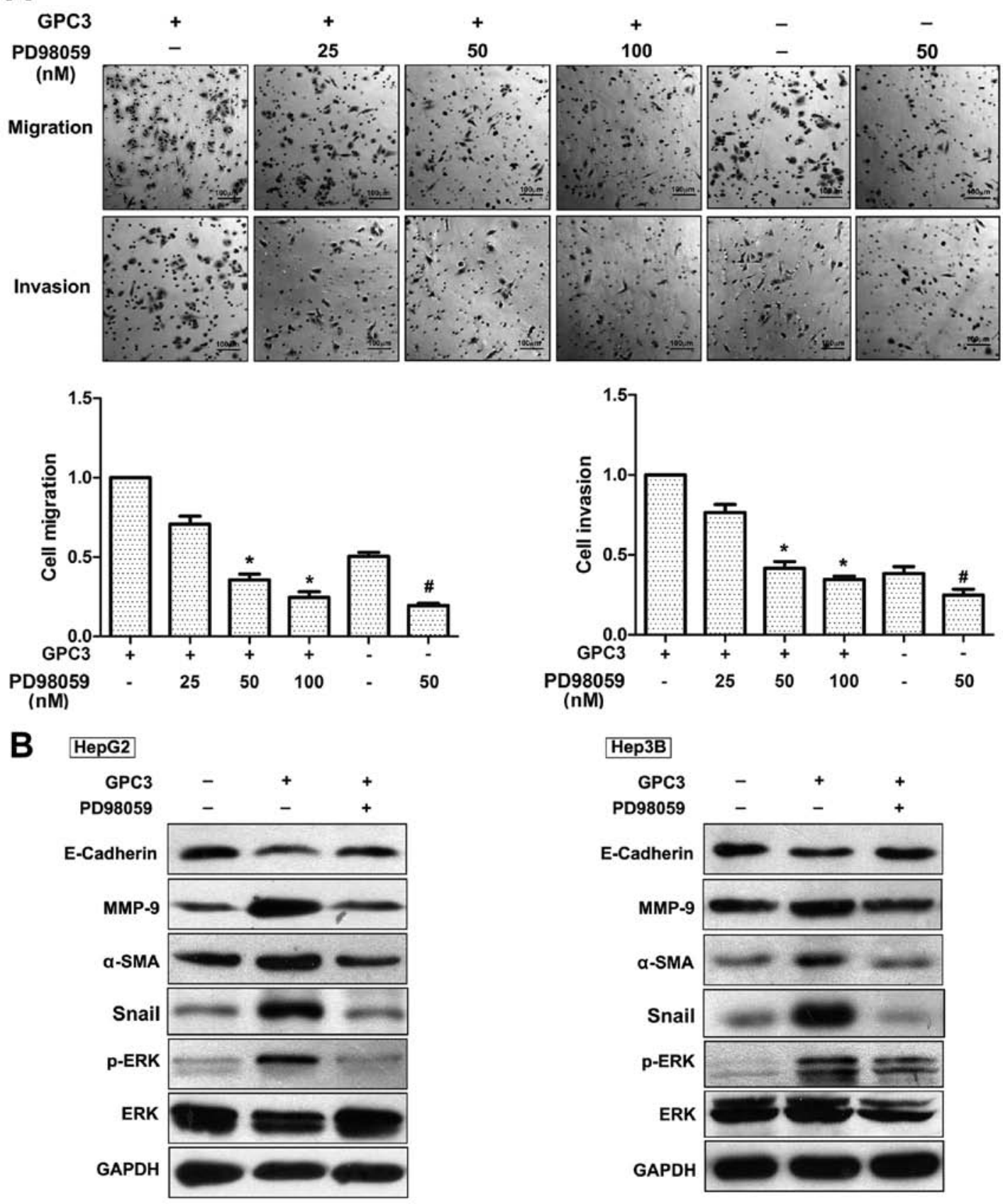

Figure 6. ERK signaling pathway was involved in GPC3-induced metastasis and EMT in HCC cells. (A) PD98059, an inhibitor of ERK, could significantly attenuate the GPC3-induced migration and invasion of HepG2 cells. Cells treated only with GPC3 served as controls. Data are shown as means \pm SDs based on three independent experiments; " $\mathrm{P}<0.05$ vs. control cells, and ${ }^{\#} \mathrm{P}<0.05$ vs. cells without treatment. (B) PD98059 could block GPC3-mediated EMT marker alterations. Cells without treatment served as controls.

BCLC staging and invasive ability. Furthermore, we found that exogenous GPC3 could regulate the migration, invasion capacity and EMT of HCC cells by activating the ERK signaling pathway.

GPC3 plays an important role in regulation of $\mathrm{HCC}$ tumorigenesis and progression. However, the effect of GPC3 on HCC proliferation is controversial. Depending on different cellular environments, GPC3 may either promote or inhibit cell growth. For example, transfection of Huh7 and HepG2 cells with GPC3-specific siRNA effectively inhibited cell proliferation (25). Cheng et al (26) reported that NIH3T3 cells transfected with a GPC3 expression vector revealed overexpression of GPC3 and excessive cell proliferation. On the other hand, Pan et al revealed that overexpression of GPC3 in Huh7 and SK-HEP-1 cells effectively inhibited cell proliferation and cell invasion through induction of apoptosis (27). Sung et al (28) revealed that antisense-mediated knockdown of GPC3 in HepG2 cells signifcantly promoted cell growth through pathways independent of IGF2. Furthermore, Lin et al (29) reported that hepatocyte proliferation and hepatomegaly induced by phenobarbital and 1,4-bis [2-(3,5-dichloropyridyloxy)] benzene is suppressed in hepatocyte-targeted glypican-3 
transgenic mice. Our laboratory is in the process of confirming the relationship between GPC3 expression and HCC cell proliferation.

Metastasis is an important aspect of HCC progression. Several studies have reported that GPC3 can stimulate the migration, and invasion $(16,17)$ of HCC cell lines. Here, we focused on the clinical importance of GPC3 in HCC, and noted that there was a positive association between GPC3 expression and HCC metastasis progression. As metastasis or vascular invasion are major factors in the progression from BCLC-stages A and B to C, we proposed that GPC3 might be involved in $\mathrm{HCC}$ metastasis. Furthermore, we showed that both endogenous and exogenous GPC3 expression were correlated with metastasis and invasion. First, we found endogenous GPC3 expression was associate with HCC metastasis in HCC tissues and cells, which was consistent with the report from Miao et al that the knockdown of GPC3 resulted in the inhibition of cell migration and invasion in Huh7 cells (16). The migration and invasion capacities of HCC cell lines were also increased by exogenous GPC3 treatment, suggesting that in addition to endogenous GPC3, soluble/exogenous GPC3 may also function to promote HCC metastasis in patients. So far, few studies have explored how endogenous and exogenous GPC3 regulate HCC metastasis. Capurro et al reported that GPC3 was needed for attachment to the cell surface by a GPI anchor to stimulate growth of HCC cells (6). Thus, we suspected that the GPI anchor is necessary in endogenous and exogenous GPC3-promoting HCC metastasis. Further study will be carried out using genetic approaches in the cell models such as loss of function studies.

The importance of the EMT in cancer metastasis and other human diseases has been recognized (30). A key step in the EMT is the downregulation of E-cadherin (31). The E-cadherin-catenin complex plays an important role in the process of cell adhesion. Its dysfunction has been associated with a reduction in cell differentiation and increased cell invasiveness and metastasis. Snail, a negative regulator of E-cadherin, is one of the key transcription factors promoting EMT (32). Furthermore, the mesenchymal markers MMP9 and $\alpha$-SMA have been particularly associated with tumor progression and metastatic dissemination in different human cancers $(33,34)$. Here, we demonstrated that GPC3 promoted tumor metastasis through the induction of EMT and could potentially be used as a biomarker. Unexpectedly, $\beta$-catenin, an epithelial cell marker, was positively correlated with GPC3 expression levels. We believe that these contradictory results are a consequence of the dual function of $\beta$-catenin in HCC. It acts in both cadherin-based adhesion and Wnt signaling. Catenins can associate with cadherins at the membrane level, forming adhesion complexes. Additionally, in response to a Wnt stimulus or specific gene mutations, $\beta$-catenin is stabilized and translocates to the nucleus where it binds $\mathrm{TCF} / \mathrm{LEF}-1$ transcription factors to trans-activate genes that drive tumor formation. Here, we measured the total levels of $\beta$-catenin. Additional studies will be needed to confirm the localization and function of $\beta$-catenin in GPC3-induced metastasis.

Many reports have shown that GPCs regulated various signaling pathways, including ERK, Shh, Wnt, bone morphogenetic proteins, and fibroblast growth factors $(5-7,26)$. We found GPC3 regulates EMT and metastasis through the ERK signaling pathway. Recent studies indicate that ERK signaling pathway is involved in Claudin-1 (35) and $\alpha \mathrm{B}-$ Crystallin $\zeta$ (36) induces EMT in HCC. Yoshida et al also reported that sublethal heat treatment skewed HCC cells toward EMT transforming them to progenitor-like by the ERK pathway (37). Thus, we believe that ERK pathway may play an important role in GPC3-induced EMT of HCC. Chen et al reported that the EMT and enhanced hedgehog signaling activity may be responsible for HCC cell chemoresistance and invasion (24). In this study, although Shh expression had an increasing trend in BCLC stage C, no significance was obtained among different stages. In addition, GPC3 treatment did not markedly increase the Shh expression in vitro. Therefore, we speculate the Shh pathway may be not involved in GPC3-induced EMT in HCC cell. Nevertheless, we cannot exclude the possibility that alternative pathways are involved in the promotion of HCC progression by GPC3. For example, GPC3 could also stimulate the in vitro and in vivo growth of HCC cells by increasing autocrine/paracrine canonical Wnt signaling (6). So identifying the precise mechanisms and signal pathways involved in these processes remains to be addressed.

In conclusion, our findings suggest that GPC3 stimulates the invasiveness and metastasis of HCC cells. GPC3 may promote the EMT of HCC cells through ERK signaling pathways. Thus, GPC3 might be a potential indicator of HCC EMT and metastasis, and our findings provide a theoretical basis for therapeutically targeting GPC3 for the treatment of HCC.

\section{Acknowledgements}

This study was supported by the Major Projects on Infectious Disease (2012ZX1002-008-05), as well as the Capital Science and Technology Development Fund (2014-1-1824) and the Beijing High-Level Talent Academic Leader/Personnel Training Programs awarded to Hui-Guo Ding (2011-2-19).

\section{References}

1. He J, Gu D, Wu X, et al: Major causes of death among men and women in China. N Engl J Med 353: 1124-1134, 2005.

2. Farazi PA and DePinho RA: Hepatocellular carcinoma pathogenesis: from genes to environment. Nat Rev Cancer 6: 674-687, 2006.

3. Huber MA, Kraut $\mathrm{N}$ and Beug $\mathrm{H}$ : Molecular requirements for epithelial-mesenchymal transition during tumor progression. Curr Opin Cell Biol 17: 548-558, 2005.

4. Kreuger J, Perez L, Giraldez AJ and Cohen SM: Opposing activities of Dally-like glypican at high and low levels of Wingless morphogen activity. Dev Cell 7: 503-512, 2004.

5. Filmus J, Capurro M and Rast J: Glypicans. Genome Biol 9: 224, 2008.

6. Capurro MI, Xiang YY, Lobe C and Filmus J: Glypican-3 promotes the growth of hepatocellular carcinoma by stimulating canonical Wnt signaling. Cancer Res 65: 6245-6254, 2005.

7. Stigliano I, Puricelli L, Filmus J, Sogayar MC, Bal de Kier Joffe E and Peters MG: Glypican-3 regulates migration, adhesion and actin cytoskeleton organization in mammary tumor cells through Wnt signaling modulation. Breast Cancer Res Treat 114: 251-262, 2009.

8. Peters MG, Farias E, Colombo L, Filmus J, Puricelli L and Bal de Kier Joffe E: Inhibition of invasion and metastasis by glypican-3 in a syngeneic breast cancer model. Breast Cancer Res Treat 80: 221-232, 2003.

9. Aviel-Ronen S, Lau SK, Pintilie M, et al: Glypican-3 is overexpressed in lung squamous cell carcinoma, but not in adenocarcinoma. Mod Pathol 21: 817-825, 2008. 
10. Capurro M, Wanless IR, Sherman M, et al: Glypican-3: a novel serum and histochemical marker for hepatocellular carcinoma Gastroenterology 125: 89-97, 2003.

11. Filmus $\mathbf{J}$ and Capurro M: Glypican-3 and alphafetoprotein as diagnostic tests for hepatocellular carcinoma. Mol Diagn 8 207-212, 2004

12. Liu H, Li P, Zhai Y, et al: Diagnostic value of glypican-3 in serum and liver for primary hepatocellular carcinoma. World $J$ Gastroenterol 16: 4410-4415, 2010.

13. Li B, Liu H, Shang HW, Li P, Li N and Ding HG: Diagnostic value of glypican-3 in alpha fetoprotein negative hepatocellular carcinoma patients. Afr Health Sci 13: 703-709, 2013.

14. Llovet JM, Chen Y, Wurmbach E, et al: A molecular signature to discriminate dysplastic nodules from early hepatocellula carcinoma in HCV cirrhosis. Gastroenterology 131: 1758-1767, 2006.

15. Wang XY, Degos F, Dubois S, et al: Glypican-3 expression in hepatocellular tumors: diagnostic value for preneoplastic lesions and hepatocellular carcinomas. Hum Pathol 37: 1435$1441,2006$.

16. Miao HL, Pan ZJ, Lei CJ, et al: Knockdown of GPC3 inhibits the proliferation of Huh7 hepatocellular carcinoma cells through down-regulation of YAP. J Cell Biochem 114: 625-631, 2013.

17. Zittermann SI, Capurro MI, Shi W and Filmus J: Soluble glypican 3 inhibits the growth of hepatocellular carcinoma in vitro and in vivo. Int J Cancer 126: 1291-1301, 2010.

18. Bruix J, Sherman M and Practice Guidelines Committee AAftSoLD: Management of hepatocellular carcinoma. Hepatology 42: 1208-1236, 2005

19. Wu YL, Wang NN, Gu L, Yang HM, Xia N and Zhang H: The suppressive effect of metabotropic glutamate receptor 5 (mGlu5) inhibition on hepatocarcinogenesis. Biochimie 94: 2366-2375, 2012.

20. Vauthey JN, Lauwers GY, Esnaola NF, et al: Simplified staging for hepatocellular carcinoma. J Clin Oncol 20: 1527-1536, 2002

21. Thiery JP, Acloque H, Huang RY and Nieto MA: Epithelialmesenchymal transitions in development and disease. Cell 139: 871-890, 2009

22. Ding W, You H, Dang H, et al: Epithelial-to-mesenchymal transition of murine liver tumor cells promotes invasion. Hepatology 52: 945-953, 2010.

23. Zhang W, Mendoza MC, Pei X, et al: Down-regulation of CMTM8 induces epithelial-to-mesenchymal transition-like changes via c-MET/extracellular signal-regulated kinase (ERK) signaling. J Biol Chem 287: 11850-11858, 2012.

24. Chen X, Lingala S, Khoobyari S, Nolta J, Zern MA and Wu J: Epithelial mesenchymal transition and hedgehog signaling activation are associated with chemoresistance and invasion of hepatoma subpopulations. J Hepatol 55: 838-845, 2011.
25. Sun CK, Chua MS, He J and So SK: Suppression of glypican 3 inhibits growth of hepatocellular carcinoma cells through up-regulation of TGF-beta2. Neoplasia 13: 735-747, 2011.

26. Cheng W, Tseng CJ, Lin TT, et al: Glypican-3-mediated oncogenesis involves the Insulin-like growth factor-signaling pathway. Carcinogenesis 29: 1319-1326, 2008.

27. Pan Z, Chen C, Long H, et al: Overexpression of GPC3 inhibits hepatocellular carcinoma cell proliferation and invasion through induction of apoptosis. Mol Med Rep 7: 969-974, 2013.

28. Sung YK, Hwang SY, Farooq M, Kim JC and Kim MK: Growth promotion of HepG2 hepatoma cells by antisense-mediated knockdown of glypican-3 is independent of insulin-like growth factor 2 signaling. Exp Mol Med 35: 257-262, 2003.

29. Lin CW, Mars WM, Paranjpe S, et al: Hepatocyte proliferation and hepatomegaly induced by phenobarbital and 1,4-bis [2-(3,5-dichloropyridyloxy)] benzene is suppressed in hepatocyte-targeted glypican 3 transgenic mice. Hepatology 54: 620-630, 2011

30. Acloque H, Adams MS, Fishwick K, Bronner-Fraser M and Nieto MA: Epithelial-mesenchymal transitions: the importance of changing cell state in development and disease. J Clin Invest 119: 1438-1449, 2009.

31. Thiery JP and Sleeman JP: Complex networks orchestrate epithelial-mesenchymal transitions. Nat Rev Mol Cell Biol 7: 131-142, 2006.

32. Zhu M, Yin F, Fan X, et al: Decreased TIP30 promotes Snailmediated epithelial-mesenchymal transition and tumor-initiating properties in hepatocellular carcinoma. Oncogene: Mar 31, 2014 (Epub ahead of print). doi: 10.1038/onc.2014.73.

33. Cho NH, Shim HS, Rha SY, et al: Increased expression of matrix metalloproteinase 9 correlates with poor prognostic variables in renal cell carcinoma. Eur Urol 44: 560-566, 2003.

34. Li H, Wang H, Wang F, Gu O and Xu X: Snail involves in the transforming growth factor beta1-mediated epithelial-mesenchymal transition of retinal pigment epithelial cells. PLoS One 6 : e23322, 2011.

35. Suh Y, Yoon CH, Kim RK, et al: Claudin-1 induces epithelialmesenchymal transition through activation of the c-Abl-ERK signaling pathway in human liver cells. Oncogene 32: 4873-4882, 2013.

36. Huang XY, Ke AW, Shi GM, et al: alphaB-crystallin complexes with 14-3-3zeta to induce epithelial-mesenchymal transition and resistance to sorafenib in hepatocellular carcinoma. Hepatology 57: 2235-2247, 2013

37. Yoshida S, Kornek M, Ikenaga N, et al: Sublethal heat treatment promotes epithelial-mesenchymal transition and enhances the malignant potential of hepatocellular carcinoma. Hepatology 58: $1667-1680,2013$ 